\title{
The CAPP II trial of aspirin in Lynch syndrome/HNPCC: is it time for everyone to be treated?
}

\author{
Patrick M. Lynch ${ }^{1}$ (1)
}

Received: 17 September 2020 / Accepted: 22 October 2020 / Published online: 9 January 2021

(c) Springer Nature B.V. 2021

In a recent issue of the Lancet, John Burn and collaborators from the CAPP II consortium, published long-term outcomes data from that trial [1]. This comprised up to 20 years of follow-up of patients, mainly a subset from the UK and Finland, who had received aspirin at a dose of $600 \mathrm{mg} / \mathrm{day}$ for an average of 25 months, or placebo.

As many Familial Cancer readers know, short term data on adenoma incidence during the period of active intervention showed no ASA benefit in reducing adenoma incidence [2]. But the CAPP II team stayed together and was able to track participants in an observation-only follow-up phase of the study. They were able to later show, for those in the aspirin arm, a significant reduction in long-term colorectal and other Lynch syndrome/HNPCC-spectrum cancers [3].

From this most recent update of the CAPP II data, key findings included: (a) a significant decrease in incident colorectal cancer was evident in Lynch syndrome carriers assigned to the aspirin arm of the trial $600 \mathrm{mg} / \mathrm{day}$. According to the per-protocol analysis the HR was 0.56 (95\% CI 0.34-0.91). Addressing a criticism of the 2011 report, the more complete dataset was also significant according to the more conservative intention-to-treat analysis, with an HR of 0.65 (95\% CI 0.43-0.97). (b) This benefit was not seen until 5 years after randomization, supporting the notion of a delay in the effect of aspirin, as seen in other studies; (c) a legacy effect was seen in which carriers taking daily aspirin for the relatively short study period of 25 months experienced a durable reduction in colorectal cancer incidence, an effect that lasted for 20 years. These findings were summarized in an accompanying Lancet editorial by Yurgelun and Chan, who suggested these most recent findings offer a "compelling rationale for broader aspirin use in most Lynch syndrome carriers" [4].

Patrick M. Lynch

plynch@mdanderson.org

1 University of Texas, Houston, TX, USA
The initial 2011 signal of a long-term cancer reduction benefit, corroborated by the longer-term UK/Finnish and conservative ITT analysis in the recent Lancet, taken together, state the case for thoughtful perseverance to completion of the ongoing CAPP III trial. This mainly UK noninferiority trial is intended primarily to determine whether lower ASA doses of $300 \mathrm{mg} /$ day or even $100 \mathrm{mg} /$ day are as effective as $600 \mathrm{mg} /$ day in reducing cancer in MMR carriers, and if so, whether with an even more acceptable safety profile.

A good study always raises several new questions for each one that it answers. CAPP II is no exception, and a number of issues arising from it will be also be considerations in viewing CAPP III. Others are broader themes having to do with chemoprevention trial design in general as well as in clinical decision-making.

Some specific issues and cautions in CAPP II are worthy of emphasis. Following completion of the planned intervention period in CAPP II there had to have been an un-blinding of patients as to their allocation to ASA or to placebo. During the longer-term observation period patients from both arms would have been at liberty to take aspirin. Once the long-term cancer-reducing benefit became evident in the 2011 report, one would imagine a tendency for subjects to resume, if in the ASA arm, or to initiate, if in the placebo arm, aspirin prophylaxis, whether at $600 \mathrm{mg} /$ day or at some other dose. If both groups of patients did adopt ASA prophylaxis on their own, then the less the long-term difference in cancer rates would be expected to be at 20 years. Yet, the observed "legacy" effect reported in this most recent Lancet paper. Collection of data on ad hoc ASA usage by CAPP II participants over the years was not systematically undertaken (John Burn, personal communication). Even if purely qualitative, subjective, and with all the biases of recall, an effort to survey CAPP II subjects on such drop-in aspirin use would be informative from a behavioral standpoint: do trial-savvy patients like what they see in terms of reported ASA benefic and choose to self-medicate, and if not, why not? Additionally, if only those originally in 
the ASA arm tended to continue aspirin then maybe the effect was not solely that of the initial treatment interval.

Long-term ASA use and in particular monitoring of adverse events among CAPP II participants would offer insights into the key question of toxicity, as it seems to most observers that a key rationale for CAPP III was to resolve nagging concerns about possible toxicity at the relatively high dose of $600 \mathrm{mg} /$ day. CAPP III is intended to determine whether or not lower doses of ASA (100 mg/day or $300 \mathrm{mg} /$ day) are as or nearly as effective as the $600 \mathrm{mg}$ /day dose employed in CAPP II. If long-term aspirin benefit is again seen in CAPP III as it was in CAPP II, at any dose, then a pretty compelling case will have been made for adopting aspirin as a standard of care in LS patients. This of course does assume no significant toxicity is seen, which if present may simply call for cautionary notes for those at increased risk of side-effects, most likely older patients.

There are other questions. Are there potentially more effective/safer NSAIDs? Naproxyn has been shown to activate immune markers in the mucosa of HNPCC/Lynch patients [5], but would it, even if effective, carry its own toxicity concerns? Would there be benefit to be had in combining aspirin with other agents? Inclusion of resistant starch in the original, more complex, factorial design of CAPP II was challenged by nonadherence. Calcium, somewhat protective against adenomas in the sporadic adenoma setting, was thought to be an agent that might potentiate aspirin, but a trial in sporadic adenomas showed no such combinatorial benefit [6].

In supplemental tables to the recent Lancet paper, the reader is reminded that the mean age at study initiation was 44 years. One tantalizing question is whether initiation of ASA much younger, say at 20-30 when colonoscopy surveillance initiation is recommended, might have had an even greater impact on cumulative CRC and other cancer incidence. On the other hand, the subjects were, on average, old enough that if a significant aspirin toxicity effect were to be seen, it would have been evident. The larger challenge is the safety of aspirin usage in Lynch syndrome/HNPCC patients who are living with colorectal cancer risk into their 70 s and 80 s. Here one must rely on the much larger studies that have been conducted in other settings. A recent cautionary note comes from a large trial in which patients over 70 were randomly assigned to low-dose $(100 \mathrm{mg} /$ day $)$ aspirin or placebo, with demonstration of significantly greater bleeding, without reduction in cardiovascular event rates [7], and higher all-cause mortality, including cancer mortality [8].

\section{Implications for patient management}

For the sake of argument let us suppose that all of the potential benefits from CAPP II are true: lasting benefit in CRC risk reduction from an ASA dose of $600 \mathrm{mg} / \mathrm{day}$, taken for at least 2-3 years in mid-adult life. And supposing further that the potential for lower dose efficacy is demonstrated in CAPP III, with acceptable toxicity for most patients. What other issues remain to be considered in managing the average patient with Lynch syndrome/HNPCC?

Many considerations are behavioral. Because cancer risk to MLH1 and MSH2 carriers begins in the early 20s, patients may need to begin dosing in their late teens, or at least 5 years before initiating colonoscopy surveillance. If this is done, will providers feel comfortable recommending longer surveillance intervals, say 3 years if conservative, 5 years if more cavalier? The PLSD data on MMR carriers, most of whom were not enrolled in CAPP II or otherwise taking aspirin as far as we know, suggests that cancer risk reduction is not improved by more frequent surveillance intervals [9]. Do we need an additional trial, randomized or observational, in which patients taking aspirin are followed at longer intervals in order to determine whether, all things considered, aspirin use safely allows a longer interval? Finally, behavioral scientists will need to tell us whether patient enthusiasm for aspirin correlates with a broader health-consciousness that tends to be associated with a desire for regular colon surveillance as well as with healthier lifestyle otherwise. Perhaps for every such patient there is another who will choose to take aspirin as an "easy fix" precisely because they don't want to go to the trouble of eating well, exercising regularly, stopping smoking, or having regular colonoscopy. One suspects the former group to be more capable of longterm adherence with a daily aspirin regimen than the latter, but who knows. Let's study it and see.

\section{References}

1. Burn J, Sheth H, Elliott F, Reed L, Macrae F, Mecklin JP et al (2020) Cancer prevention with aspirin in hereditary colorectal cancer (Lynch syndrome), 10-year follow-up and registry-based 20-year data in the CAPP2 study: a double-blind, randomised, placebo-controlled trial. Lancet 395(10240):1855-1863

2. Burn J, Bishop DT, Mecklin JP, Macrae F, Moslein G, Olschwang $S$ et al (2008) Effect of aspirin or resistant starch on colorectal neoplasia in the Lynch syndrome. N Engl J Med 359(24):2567-2578

3. Burn J, Gerdes AM, Macrae F, Mecklin JP, Moeslein G, Olschwang $\mathrm{S}$ et al (2011) Long-term effect of aspirin on cancer risk in carriers of hereditary colorectal cancer: an analysis from the CAPP2 randomised controlled trial. Lancet 378(9809):2081-2087

4. Yurgelun MB, Chan AT (2020) Aspirin for Lynch syndrome: a legacy of prevention. Lancet 395(10240):1817-1818

5. Reyes-Uribe L, Wu W, Gelincik O, Bommi PV, Francisco-Cruz A, Solis LM et al (2020) Naproxen chemoprevention promotes immune activation in Lynch syndrome colorectal mucosa. Gut 69(10):1-12

6. Pommergaard HC, Burcharth J, Rosenberg J, Raskov H (2016) Aspirin, calcitriol, and calcium do not prevent adenoma recurrence in a randomized controlled trial. Gastroenterology. 150(1):114-122.e4 
7. McNeil JJ, Wolfe R, Woods RL, Tonkin AM, Donnan GA, Nelson MR et al (2018) Effect of aspirin on cardiovascular events and bleeding in the healthy elderly. N Engl J Med 379(16):1509-1518

8. McNeil JJ, Nelson MR, Woods RL, Lockery JE, Wolfe R, Reid $\mathrm{CM}$ et al (2018) Effect of aspirin on all-cause mortality in the healthy elderly. N Engl J Med 379(16):1519-1528

9. Engel C, Vasen HF, Seppala T, Aretz S, Bigirwamungu-Bargeman M, de Boer SY et al (2018) No difference in colorectal cancer incidence or stage at detection by colonoscopy among 3 countries with different lynch syndrome surveillance policies. Gastroenterology. 155(5):1400-1409.e2

Publisher's Note Springer Nature remains neutral with regard to jurisdictional claims in published maps and institutional affiliations. 